\title{
TransCon CNP, a Sustained-Release C-Type Natriuretic Peptide Prodrug, a Potentially Safe and Efficacious New Therapeutic Modality for the Treatment of Comorbidities Associated with Fibroblast Growth Factor Receptor 3-Related Skeletal Dysplasias
}

\author{
Vibeke Miller Breinholt, Caroline E. Rasmussen, Per Holse Mygind, Mads Kjelgaard-Hansen, \\ Frank Faltinger, Ana Bernhard, Joachim Zettler, and Ulrich Hersel \\ Ascendis Pharma A/S, Hellerup, Denmark (V.M.B., C.E.R., P.H.M., M.K.-H.); and Ascendis Pharma GmbH, Heidelberg, Germany \\ (F.F., A.B., J.Z., U.H.)
}

Received March 25, 2019; accepted June 18, 2019

\begin{abstract}
TransCon CNP is a C-type natriuretic peptide (CNP-38) conjugated via a cleavable linker to a polyethylene glycol carrier molecule, designed to provide sustained systemic CNP levels upon weekly subcutaneous administration. TransCon CNP is in clinical development for the treatment of comorbidities associated with achondroplasia. In both mice and cynomolgus monkeys, sustained exposure to CNP via TransCon CNP was more efficacious in stimulating bone growth than intermittent CNP exposure. TransCon CNP was well tolerated with no adverse cardiovascular effects observed at exposure levels exceeding the expected clinical therapeutic exposure. At equivalent dose levels, reductions in blood pressure and/or an increase in heart rate were seen following single subcutaneous injections of the unconjugated CNP-38 molecule or a daily CNP-39 molecule (same amino acid sequence as Vosoritide, USAN:INN). The half-life of the daily CNP-39 molecule in cynomolgus monkey was estimated to be 20 minutes, compared with 90 hours for CNP-38, released from TransCon CNP. $C_{\max }$ for the CNP-39 molecule $(20 \mu \mathrm{g} / \mathrm{kg})$ was approximately 100 -fold higher, compared with the peak CNP level associated with administration of $100 \mu \mathrm{g} / \mathrm{kg}$ CNP as TransCon CNP. Furthermore, CNP
\end{abstract}

exposure for the daily CNP-39 molecule was only evident for up to 2 hours postdose (lower limit of quantification $37 \mathrm{pmol} / \mathrm{l}$ ), whereas TransCon CNP gave rise to systemic exposure to CNP-38 for at least 7 days postdose. The prolonged CNP exposure and associated hemodynamically safe peak serum concentrations associated with TransCon CNP administration are suggested to improve efficacy, compared with short-lived CNP molecules, due to better therapeutic drug coverage and decreased risk of hypotension.

\section{SIGNIFICANCE STATEMENT}

The hormone C-type natriuretic peptide (CNP) is in clinical development for the treatment of comorbidities associated with achondroplasia, the most common form of human dwarfism. The TransCon Technology was used to design TransCon CNP, a prodrug that slowly releases active CNP in the body over several days. Preclinical data show great promise for TransCon CNP to be an effective and well-tolerated drug that provides sustained levels of CNP in a convenient once-weekly dose, while avoiding high systemic CNP bolus concentrations that can induce cardiovascular side effects.

\section{Introduction}

Currently, no approved treatment exists for comorbidities associated with achondroplasia (ACH), the most common form of human dwarfism. Administration of C-type natriuretic

This work was supported by Ascendis Pharma, where V.M.B., C.E.R., P.H.M., and M.K.-H. are employees of Ascendis Pharma A/S, and U.H., J.Z., F.F., and A.B. are employees of Ascendis Pharma GmbH, and have received cash and equity compensation from Ascendis Pharma during their employment.

https://doi.org/10.1124/jpet.119.258251. peptide (CNP) to ACH patients and animals has been shown to stimulate endochondral bone growth and improve adverse skeletal manifestations of the disease (Yasoda et al., 2009; Lorget et al., 2012; Wendt et al., 2015; Legeai-Mallet, 2016; https://investors.biomarin.com/download/R\%26DDay_ FINAL_110618.pdf). The biology of CNP has been known for many years, but the use of CNP as a pharmaceutical product has been limited by major shortcomings of the native CNP. Native CNP has a half-life of only 2 to 3 minutes in humans, and degradation by neutral endopeptidase (NEP) makes it difficult to maintain an efficacious therapeutic drug exposure.

ABBREVIATIONS: ACH, achondroplasia; BAP, bone-specific alkaline phosphatase; BMD bone mineral density; BP, blood pressure; cGMP, cyclic guanosine monophosphate; CNP, C-type natriuretic peptide; CTX C telopeptides of type I collagen; DSI Data Sciences International; FGFR3, fibroblast growth factor receptor 3; HPLC, high-performance liquid chromatography; HPLC-MS/MS high-performance liquid chromatography with tandem mass spectrometric detection; HR, heart rate; LLOQ, lower limit of quantification; MAP, mean arterial pressure; NEP, neutral endopeptidase; NPR-B, natriuretic peptide receptor B; NPR-C, natriuretic peptide receptor C; NTx, N-terminal telopeptide; OC, osteocalcin; PEG, polyethylene glycol; PINP, procollagen type I N-terminal propeptide; PK, pharmacokinetic; QC Quality control; RIA, radioimmunoassay; SAP, systolic arterial pressure; vBMD, apparent density of the total region; VOI, volume of interest; WCX, weak cation exchange. 
Because of the short half-life of native CNP, continuous intravenous administration of CNP-22 is required to restore bone growth in ACH mice (Yasoda et al., 2009). Wendt et al. (2015) recently reported the development of a daily CNP analog, with increased NEP resistance, and an associated subcutaneous half-life in mice of about 15 minutes, compared with $<5$ minutes for murine CNP-22 (Wendt et al., 2015). A corresponding long half-life of approximately 20 minutes was verified in cynomolgus monkeys (Wendt et al., 2015). The daily CNP analog with the INN name Vosoritide consists of the $37 \mathrm{C}$-terminal amino acids of human CNP plus the amino acids glycine and proline added to the N-terminal portion of the peptide. Proof-of-principle for this daily CNP analog was obtained in the nonclinical setting employing ACH mice exhibiting phenotypical traits similar to that observed in human individuals with $\mathrm{ACH}$ (Lorget et al., 2012; Wendt et al., 2015). Daily administration of this CNP analog improved the major phenotypical dwarfism traits by normalizing chondrocyte proliferation and differentiation facilitating bone growth in both axial and appendicular bones (Lorget et al., 2012; Wendt et al., 2015).

The mechanism of action of CNP is believed to be via inhibition of the downstream signaling pathway of the fibroblast growth factor receptor 3 (FGFR3) at the level of raf, thereby restoring chondrogenesis by counteracting the overactivation of the FGFR3 pathway seen in ACH (Laederich and Horton, 2010). In children with ACH, the daily administered CNP molecule Vosoritide positively impacts bone growth, resulting in an increase in annualized height velocity of approximately 1 to $2 \mathrm{~cm} / \mathrm{y}$ (https://investors.biomarin.com/ download/R\%26DDay_FINAL_110618.pdf).

As the mutated FGFR3 receptor is constitutively overactivated, we hypothesize that continuous exposure to CNP is required to optimally counterbalance the overactivated FGFR3 signaling pathway, and that short-acting CNP molecules may suffer suboptimal efficacy due to their short half-life preventing optimal modulation of the overactive FGFR3 pathway. Another potential limitation of CNP as a therapeutic modality in ACH treatment is the vasodilatory effect of CNP (Clavell et al., 1993; Igaki et al., 1998; Chauhan et al., 2003; Scotland et al., 2005; Nakao et al., 2017). High concentrations of CNP cause vasodilation, with resulting decreased blood pressure (BP) in animals (Lopez et al., 1997; Wendt et al., 2015). Intravenous bolus administration of CNP-22 in humans induces postdose hypotension and tachycardia, coinciding with the peak blood concentration, indicating a causal relationship (Igaki et al., 1998). Decreases in BP, occurring shortly after dose administration and coincident with high CNP exposure, have also been observed in children with $\mathrm{ACH}$ following administration of Vosoritide (https://investors.biomarin. com/download/R\%26DDay_FINAL_110618.pdf).

Thus, for CNP to be both an efficacious and safe therapy for $\mathrm{ACH}$, administration should optimally provide continuous exposure to restore chondrogenesis and promote bone growth, while avoiding high systemic concentrations that can induce cardiovascular side effects.

TransCon CNP, a sustained-release prodrug of CNP, is in clinical development as a potentially safe and efficacious treatment of achondroplasia. To ensure sustained delivery, CNP is transiently bound via the TransCon Linker to a chemically inert, branched polyethylene glycol (PEG) carrier that prolongs the peptide's overall circulatory halflife. This is achieved by minimizing renal clearance of the TransCon CNP prodrug and shielding of the CNP molecule from proteolytic degradation and from binding to its primary activating and clearance receptors, natriuretic peptide receptor B (NPR-B) and natriuretic peptide receptor C (NPR-C). Following cleavage of the TransCon Linker under physiologic $\mathrm{pH}$ and temperature, active CNP38 peptide is slowly and continuously released and, due to the small size of the peptide, assumed to be subjected to the same distribution and pharmacodynamics mechanisms as endogenous CNP.

In this publication, we describe the in vitro and in vivo screenings that led to the selection and development of TransCon CNP as a promising treatment modality for ACH and potentially other skeletal dysplasias, in which CNP may impact positively on a constitutively overactivated FGFR3 pathway.

\section{Materials and Methods}

\section{Selection of TransCon CNP}

CNP-38 having the sequence LQEHPNARKYKGANKKGLSKGCFGLKLDRIGSMSGLGC (oxidized to form one intramolecular disulfide bond), fluorescein-labeled CNP-38, and CNP-38 conjugates (Table 1) used for the in vivo and the in vitro studies were synthesized chemically, as described in patent applications (Sprogøe et al., 2016; Rau et al., 2017).

Deuterated CNP-38 was used as internal standard for the free CNP analysis and synthesized as described for CNP-38, with the exception that deuterated leucine (D10 Fmoc-Leu-OH; Cambridge Isotope Laboratories) was incorporated at positions Leu1, Leu25, and Leu36. The daily CNP-39 molecule with the sequence PGQEHPNARKYKGANKKGLSKGCFGLKLDRIGSMSGLGC (oxidized to form one intramolecular disulfide bond) was synthesized chemically as described for CNP-38. This peptide thus consists of the amino acids proline (amino acid 1) and glycine (amino acid 2) plus the 37 C-terminal amino acids of natural human CNP. This resulting peptide is similar to Vosoritide, which is in clinical development for the treatment of comorbidities associated with achondroplasia (https:// investors.biomarin.com/download/R\%26DDay_FINAL_110618.pdf), and in this document referred to as the daily CNP-39 molecule.

\section{Activity (Cyclic Guanosine Monophosphate) Assay in NIH3T3 Cells}

The activity of the CNP-38 conjugates to elicit an intracellular cyclic guanosine monophosphate (cGMP) response was determined in NIH3T3 cells. These cells express NPR-B on the cell surface (Abbey and Potter, 2003), and stimulation of this receptor with CNP leads to intracellular production of the secondary messenger cGMP. In brief, NIH3T3 cells were cultured in Dulbecco's modified Eagle's medium F-12 medium with 5\% FBS and $5 \mathrm{mM}$ glutamine at $37^{\circ} \mathrm{C}$ and $5 \% \mathrm{CO}_{2}$. For each assay, cells were resuspended in stimulation buffer (Dulbecco's PBS with $0.5 \mathrm{mM}$ 3-isobutyl-1methylxanthine), seeded in a 96 -well plate $\left(5 \times 10^{4} /\right.$ well), and incubated in duplicate with the CNP conjugate at different concentrations. After an incubation of 30 minutes at $37^{\circ} \mathrm{C}$ and $5 \% \mathrm{CO}_{2}$, cells were lysed in the supplied lysis buffer, and the cGMP level was measured using a commercially available cGMP assay based on time-resolved fluorescence energy transfer (cGMP kit, catalogue 62GM2PEB; Cisbio, Codolet, France). Activity was determined using a four-parameter logistic curve fit and by parallel line analysis of the sample in comparison with CNP-38 as 
a reference standard (PLA 2.0 software; Stegmann Systems, Rodgau, Germany).

\section{NPR-C Affinity Assay}

For the NPR-C affinity assay, a HEK293 cell line, stably overexpressing human NPR-C, was developed. The HEK293 ${ }^{\text {NPR-C }}$ cell line was developed as follows: the coding region of human NPR-C reference sequence was cloned into a lentiviral vector under a cytomegalovirus promoter to allow for constitutive receptor expression. A bicistronic element located on the vector for puromycin resistance was used as the eukaryotic selection marker. After transduction, pools of stably growing cells were subjected to quantitative reverse-transcription polymerase chain reaction for confirmation of NPR-C mRNA expression. High receptor mRNA expression was confirmed as compared with parental HEK293. For NPR-C affinity analysis, cells were routinely cultured in Dulbecco's modified Eagle's medium/Glutamax medium with $10 \% \mathrm{FBS}$ and $0.5 \mu \mathrm{g} / \mathrm{ml}$ puromycin at $37^{\circ} \mathrm{C}$ and $5 \% \mathrm{CO}_{2}$. Growing cells were trypsinized from the cell flask bottom, counted, seeded in a 96 -well plate $\left(1.5 \times 10^{5} /\right.$ well) (U-bottom wells; Greiner BioOne, Frickenhausen, Germany), and centrifuged. The supernatants were discarded. CNP-38 standards and samples were serially diluted over nine steps in PBS $0.2 \%$ bovine serum albumin, transferred to duplicate microplates, and mixed with the cell pellet. After 30 -minute incubation at room temperature, fluorescein-labeled CNP-38 was added to each well at a fixed concentration, and cells were incubated for additional 45 minutes at room temperature. Subsequently, cells were analyzed by flow cytometry using the mean fluorescence intensity of the fluorescein isothiocyanate channel as the readout. The CNP-38 standard and sample curves were generated in parallel line analysis software (PLA 2.0; Stegmann Systems) using a fourparameter logistic fit for calculation of the relative NPR-C affinity of sample versus the CNP-38 standard (ratio $\mathrm{IC}_{50} \mathrm{CNP}_{-38 / \mathrm{IC}_{50} \mathrm{CNP}-38}$ conjugate).

\section{NEP Resistance}

The degradation half-life of various CNP conjugates was measured in a NEP digestion assay. Recombinant human NEP $(2.5 \mu \mathrm{g} / \mathrm{ml}$ final concentration, BML-SE532-0010; Enzo Life Science) and the internal standard pentafluorophenol ( $40 \mu \mathrm{g} / \mathrm{ml}$ final concentration) were added to a solution of the CNP conjugate $(100 \mu \mathrm{g}$ CNP equivalents $/ \mathrm{ml})$ contained in digest buffer ( $50 \mathrm{mM}$ Tris- $\mathrm{HCl}, 10 \mathrm{mM} \mathrm{NaCl}, \mathrm{pH} 7.4$ ). The solution was incubated at $37^{\circ} \mathrm{C}$ and $500 \mathrm{rpm}$ for up to 4 days in a thermomixer. Samples were removed at several time points during the 4-day incubation period. The reaction was stopped by adding Tris(2-carboxyethyl)phosphine (25 $\mathrm{mM}$ final concentration) and incubating the mixture at $95^{\circ} \mathrm{C}$, at $500 \mathrm{rpm}$, for 5 minutes. The half-life of each CNP variant was calculated using the loss of the highperformance liquid chromatography (HPLC)-UV peak area over time relative to pentafluorophenol.

\section{In Vivo Studies}

Animals and Husbandry. All animal procedures were carried out in accordance with the Guide for the Care and Use of Laboratory Animals as adopted and promulgated by the National Institutes of Health, and were approved by and performed in Association for Assessment and Accreditation of Laboratory Animal Care-accredited facilities (Charles River Laboratories, Senneville, Canada, and Envigo, Cambridgeshire, UK). The total number of animals used in the current studies was considered the minimum required to properly accomplish the objectives of the studies. All studies and procedures were conducted in accordance with the highest standards of animal care, and in compliance with federal, state, and local laws and institutional regulations.

Cardiovascular Studies. The hemodynamic tolerability of subcutaneous administered TransCon CNP and the daily CNP-39 molecule was investigated in both mice and cynomolgus monkeys, whereas unconjugated CNP-38 was only evaluated in mice.

\section{Mice}

In male telemetered adult Crl:CD1(ICR) mice, the cardiovascular evaluation of unconjugated CNP-38 and TransCon CNP included potential effects on $\mathrm{BP}$ and heart rate $(\mathrm{HR})$. Animals $(n=4)$ were dosed subcutaneously with single doses of vehicle, CNP-38, or TransCon CNP at $800 \mu \mathrm{g}$ CNP/kg. Animals were dosed with TransCon CNP and CNP-38 on different days, but always together with timematched vehicle controls. Systemic BP [systolic arterial pressure (SAP), diastolic arterial pressure, and mean arterial pressure (MAP)] and HR (derived from BP monitoring) were recorded from an implanted Data Sciences International (DSI) transmitter (DSI TA11PA-C10) using a digital data capture system linked with a DSITM Ponemah data acquisition (DSI PONEMAH 5.2) and analysis system (OpenART version 4.3). The data capture system allowed recording of the cardiovascular parameters, whereas the mice were in individual cages. On each of the dosing occasions, a predose recording of a duration of approximately 60 minutes was performed. Immediately after each dose administration, the mice were returned to their cages, and a 48-hour cardiovascular monitoring was initiated. Systemic BP and HR were reported at the following time points: $-0.5,-0.33,-0.17,0.08,0.25,0.5,1,2,6$, $12,18,24,30,36,42$, and 48 hours postdose, with each time point being the mean of 5 minutes of data. MAP measurements were tested for statistical difference to vehicle group (using Williams' test; analysis of covariance adjusted for differences between the treatment groups at baseline).

\section{Monkeys}

In adult male telemetered cynomolgus monkeys, the cardiovascular evaluation encompassed potential effects of TransCon CNP on

TABLE 1

CNP-38 conjugates used for in vivo and in vitro studies

\begin{tabular}{|c|c|}
\hline $\begin{array}{l}\text { CNP-38 Conjugate (Linker Type, Linker Attachment } \\
\text { Site) }\end{array}$ & Description \\
\hline 5-kDa PEG-linker CNP-38 (permanent, Lys26) & -kDa PEG carrier conjugated to the Lys 26 side chain of CNP-38 via a permanent linker \\
\hline $\begin{array}{l}\text { 5-kDa PEG-linker CNP-38 (permanent, } \\
\text { N-terminus) }\end{array}$ & \\
\hline $\begin{array}{l}4 \times 10 \text {-kDa PEG-linker CNP-38 (permanent, } \\
\text { Lys26) }\end{array}$ & $\begin{array}{c}\text { 4-Arm-branched } 4 \times 10-\mathrm{kDa} \text { PEG carrier conjugated to the Lys26 side chain of CNP-38 via } \\
\text { a permanent linker }\end{array}$ \\
\hline $\begin{array}{l}2 \times 20 \text {-kDa PEG-linker CNP-38 (transient, } \\
\text { Lys26) }\end{array}$ & $\begin{array}{c}\text { 2-Arm-branched } 2 \times 20-\mathrm{kDa} \text { PEG carrier conjugated to the Lys26 side chain of CNP-38 via } \\
\text { a TransCon linker }{ }^{a}\end{array}$ \\
\hline $\begin{array}{l}\mathrm{C}^{b}(10-\mathrm{kDa} \text { PEG-linker CNP-38) } \\
\text { Lys26) }\end{array}$ & $\begin{array}{l}\text { Four CNP-38 molecules conjugated to a } 4 \text {-arm-branched } 4 \times 10-\mathrm{kDa} \text { PEG; one CNP-38 is attached } \\
\text { via its Lys } 26 \text { side chain and via a permanent linker to the distal end of each of the four PEG arms }\end{array}$ \\
\hline
\end{tabular}

${ }^{a}$ The linker attachment site to the PEG carrier is close to the branching site of the PEG arms.

${ }^{b} \mathrm{C}$ represents the central carbon atom, which is the branching point of the four PEG arms. 
BP, HR, ECG, and body temperature. TransCon CNP and the daily CNP-39 molecule were administered as single subcutaneous injections in an escalating dose design ( $n=4 /$ dose level), including six test sessions. On test session 1 , all animals received vehicle. On test sessions 2 and 3, all animals received dose levels of the daily CNP-39 molecule (positive control) at 50 and $100 \mu \mathrm{g}$ CNP/kg, respectively. On test sessions 4-6, all animals received TransCon CNP at 10, 40, or $100 \mu \mathrm{g} \mathrm{CNP} / \mathrm{kg}$, respectively. A washout period of 3 days was allowed between test sessions 1 and 2; 7 days between test sessions 2, 3, and 4; and at least 14 days between test sessions 4, 5, and 6. Body temperature and the following cardiovascular parameters were recorded via a DSI transmitter implant (DSI TL11M2-D70-PCT) and using the DSI digital data capture system Ponemah Physiology Platform P3 software system (DSI PONEMAH 5.1 and 5.2, ADaPT 2.0): systemic BP (SAP, diastolic arterial pressure, MAP, and pulse pressures), HR (derived from BP), and ECG duration/intervals (PR, RR, QRS, QT, and QTca) up to 24 hours after administration of the daily CNP-39 molecule and up to 48 hours after administration of vehicle or TransCon CNP. Blood samples were collected at 48 and 168 hours after administration of TransCon CNP, to verify that animals received the correct dose.

\section{Mouse Efficacy Study: Pharmacological Effects of Daily Bolus Versus Continuous Subcutaneous Infusion of CNP-38 in Mice}

Groups ( $n=9$ ) of 3 -week-old ( \pm 1 day) male FVB mice (Charles River Laboratories) were administered $203 \mu \mathrm{g} / \mathrm{kg}$ per day CNP-38 for 5 weeks either by continuous subcutaneous infusion or by single daily subcutaneous injections. Continuous infusion was applied by osmotic Alzet pumps (Model 1002, $0.25 \mu \mathrm{l} / \mathrm{h}$ for study day 1-15; Model 1004, $0.09 \mu \mathrm{l} / \mathrm{h}$ for study day $15-34)$. CNP-38 was formulated either at $577 \mu \mathrm{g} / \mathrm{ml}$ (Model 1002, day 1), $2254 \mu \mathrm{g} / \mathrm{ml}$ (Model 1004, day 15 ), or $40.6 \mu \mathrm{g} / \mathrm{ml}$ (daily subcutaneous injection) in vehicle (30 $\mathrm{mM}$ acetate buffer, $\mathrm{pH} 4$, containing $5 \%$ sucrose and $1 \%$ benzylic alcohol). The vehicle was used as control in both the daily injection and continuous infusion groups. The pumps were implanted subcutaneously in the scapular area at day 1 (Model 1002) and were exchanged on day 15 (Model 1004) to adjust for the increased body weight and to ensure adequate dose coverage over the 5 -week infusion period. Doses for continuous infusions were calculated using an average body weight of $17 \mathrm{~g}$ at study day 7 (Model 1002) and $24 \mathrm{~g}$ at study day 25 (Model 1004), according to FVB mouse growth charts. Daily subcutaneous injections were given at the same time of the day ( \pm 2 hours) and based on the most recent body weight. The body length was measured weekly. At day 35, the mice were anesthetized, and the length of the spine (lateral view, distance from the most cranial end of L1 vertebral body to the most caudal end of the L6 vertebral body and accounting for curvature manually), the right femur (distance from the most proximal femoral head ossification center to the most distal ossification center in dorso-ventral view), and the right tibia (distance from the most proximal ossification center to the most distal ossification center in dorso-ventral view) was determined by digital X-ray (MX20; Faxitron). Treated groups were compared with corresponding vehicle control groups. Statistical analysis was performed using a one-factor ANOVA model for the logarithm of length. The four-level factor comprised all combinations of treatment (vehicle, CNP-38) and administration route (Alzet pumps, subcutaneous). The relative change from control was derived from the estimated treatment differences in the model. Data were analyzed using SAS 9.4 software (SAS institute, Cary).

Design of Cynomolgus Monkey Pharmacokinetic and Efficacy Study. Groups of four male cynomolgus monkeys were administered 0,40 , or $100 \mu \mathrm{g} / \mathrm{kg}$ TransCon CNP at 10,40 , or 100 once weekly for 26 weeks by the subcutaneous route. An additional group of four animals received the daily CNP-39 molecule (positive control), once daily for 26 weeks. The monkeys were of Chinese origin and received from RMS Houston (Houston, TX). The animals were acclimatized for 5 weeks prior to study start. The animals were $25-29$ months old and weighed between 2.0 and $3.1 \mathrm{~kg}$ at start of dosing and hence skeletally immature. Animals were socially housed (up to two animals of same sex and same dosing group together). PMI Nutrition International Certified Primate Chow No. 5048 was provided twice daily, except during designated procedures. The chow was provided in amounts appropriate for the size and age of the animals. Target temperatures of $20-26^{\circ} \mathrm{C}$ with a relative target humidity of $30 \%-70 \%$ were maintained. A 12-hour light/12-hour dark cycle was maintained, except when interrupted for designated procedures. Animals were sacrificed 7 days after the last dose administration.

Pharmacokinetics in Cynomolgus Monkey. The pharmacokinetic (PK) profile of CNP released from the TransCon CNP prodrug was investigated and compared with that of daily administered CNP39 molecule, during the time course of a 26 -week juvenile monkey efficacy study (study design described above). Blood samples for CNP38 analysis were collected and analyzed following the 1 st and the 26 th dose at the following time points: 0 (prior to dosing), $6,12,24,36,48$, $72,96,120,144$, and 168 hours postdose. Samples from animals treated with the daily CNP-39 molecule were also obtained after the 1 st and the 26th dose: at pre-dose, 0.08, 0.25, 0.5, 1, 2, and 4 hours postdose.

For analysis of free CNP-38, blood was collected into precooled lithium heparin tubes, mixed, and maintained chilled on wet ice. Within 60 seconds of collection, $250 \mu \mathrm{l}$ heparinized blood was transferred into a polypropylene tube kept on ice containing $50 \mu \mathrm{l}$ acidic citrate buffer (0.5 M sodium citrate, $\mathrm{pH} 4.0)$. After mixing, acidified plasma was generated via centrifugation at $4^{\circ} \mathrm{C}$.

Free CNP-38 was quantified after plasma precipitation, weak cation exchange (WCX) solid-phase extraction, and subsequent highperformance liquid chromatography with tandem mass spectrometric detection (HPLC-MS/MS) analysis with deuterated CNP-38 as internal standard. The standards $(150 \mu \mathrm{l})$ [calibration range 2-250 $\mathrm{pmol} / \mathrm{l}$ in blank Li-Heparin cynomolgus monkey plasma, acidified with $0.5 \mathrm{M}$ sodium citrate, pH 4.0 (5/2, v/v); LPT KG, Hamburg, Germany], the quality control samples (QCs), and the acidified monkey plasma samples $(150 \mu \mathrm{l})$ were precipitated with $150 \mu \mathrm{l} 0.2 \%$ formic acid in methanol containing the internal standard. After centrifugation at ambient temperature, the supernatant was transferred to a 96-well plate. The transferred supernatant was diluted with $100 \mu \mathrm{l} 0.01 \mathrm{M}$ methionine in water. The so far processed standards, QCs, and samples were applied to preconditioned cavities of a WCX solidphase extraction well plate (SOLA $\mu$ WCX $2 \mathrm{mg} / 1 \mathrm{ml}$, 60209-004; Thermo Scientific) and centrifuged. Washing was performed by centrifugation using $30 \mu \mathrm{l} 0.01 \mathrm{M}$ methionine in water, followed by two times $30 \mu \mathrm{l} 0.01 \mathrm{M}$ methionine in water/acetonitrile $(80 / 20, \mathrm{v} / \mathrm{v})$. Elution was performed using three times $30 \mu \mathrm{l} 11.4 \%$ trifluoroacetic acid in water/acetonitrile $[\sim 1 / 4 / 4(\mathrm{v} / \mathrm{v} / \mathrm{v})]$. The solutions were then evaporated until dryness in a stream of nitrogen. The residues were

TABLE 2

Evaluation analysis parameters for XtremeCT data

\begin{tabular}{lcrr}
\hline & Evaluation Ulna Growth Plate & Evaluation Radius Metaphysis & Evaluation Radius Diaphysis \\
\hline Sigma & 0.8 & & 0.8 \\
Support & 1 & 150 (total/cortical) and 110 (trabecular) & 1 \\
Lower threshold & 95 & 1000 & 150 \\
Upper threshold & 1000 & & 1000 \\
\hline
\end{tabular}


reconstituted with $50 \mu \mathrm{l}$ water/methanol/formic acid (11/8/1 v/v/v) and subsequently analyzed by HPLC-MS/MS. Chromatography was carried out on an Agilent 1290 Infinity (Agilent Technologies) with water plus $0.2 \%$ formic acid and acetonitrile plus $0.2 \%$ formic acid as eluents with postcolumn infusion of $0.25 \% \mathrm{DMSO}$ in water as supercharger. MS/MS analysis was done with selected MRM transitions for CNP-38 (ESI, positive mode, $\mathrm{m} / \mathrm{z} 581.1$ to 656.2 ) and the deuterated internal standard (m/z 585.4 to659.4) on an AbSciex $6500^{+}$QTrap (AbSciex, Darmstadt, Germany). The concentration of free CNP in study samples was calculated from a calibration curve (linear regression, $1 / \mathrm{x}^{2}$ weighted). The LLOQ of the assay was $2 \mathrm{pmol} / \mathrm{l}$.

The daily CNP-39 molecule was measured using a commercially available competitive radioimmunoassay (RKU-012-03; Phoenix Pharmaceuticals). The protocol provided by the manufacturer was used to qualify the assay using Li-Heparin plasma from cynomolgus monkey. All samples $(20 \mu \mathrm{l})$ were diluted in $180 \mu \mathrm{l}$ radioimmunoassay (RIA) buffer supplied and incubated with the supplied rabbit antiCNP antibody $(100 \mu \mathrm{l})$ for 5 hours at room temperature. All samples were subsequently incubated with the supplied iodine tracer $(100 \mu \mathrm{l})$, and immunoprecipitation was carried out for 16 to 24 hours at $5^{\circ} \mathrm{C}$. On day 2, $100 \mu \mathrm{l}$ goat anti-rabbit IgG serum and $100 \mu \mathrm{l}$ normal rabbit serum were added, and samples were further incubated for 90 minutes at room temperature. Upon incubation, $500 \mu \mathrm{l}$ RIA buffer was added, and the sample was centrifuged for 20 minutes at $3000 \mathrm{rpm}\left(4^{\circ} \mathrm{C}\right)$. The supernatant was discarded by tube inversion. All tubes were immediately placed in a $\gamma$ counter and measured for 5 minutes. Quantitation of study samples was carried out over an analytical range of $150-1080 \mathrm{pg} / \mathrm{ml}$, using calibration standards of the daily CNP-39 molecule $(75 ; 150 ; 300 ; 450 ; 600 ; 750 ; 900 ; 1080 \mathrm{pg} / \mathrm{ml})$ and quality controls $(250 ; 400 ; 800 \mathrm{pg} / \mathrm{ml})$. Samples were analyzed across 10 analytical runs. Assay coefficient of variation (at each QC level) was below $20 \%$. The quantification was performed using the radioactivity of the sample expressed in cpm. The calibration curve was fitted by nonlinear regression using the four-parameter logistic function (Watson/LIMS: logistic autoestimate, no weighting).

The PK evaluation was performed by noncompartmental analysis using the Phoenix WinNonlin Software.

Bone Markers. A panel of musculoskeletal endpoints was assessed during the 26-week treatment period, including biochemical markers of bone turnover [bone formation markers: bone-specific alkaline phosphatase (BAP); MicroVueTM BAP Enzyme Immunoassay kit (catalogue 8012; Quidel), procollagen type I N-terminal propeptide (PINP); UniQ PINP RIA Kit (catalogue 67034; Orion Diagnostica), osteocalcin (OC); MicroVue Osteocalcin Enzyme Immunoassay kit (catalogue 8001; Quidel) in serum, as well as the bone resorption markers: C telopeptides of type I collagen (serum CTx), ELISA kit (catalogue: AC-02F1; Immunodiagnostic Systems) in serum, and N-terminal telopeptide (NTx); Osteomark NTx Urine ELISA kit (catalogue X9006 or 9006; Abbott) in urine].

For the biochemical markers of bone turnover, animals were fasted overnight before blood sampling. Blood samples were collected between 08:00 and 10:00 AM prior to dosing and processed to serum. Animals were deprived of food during the urine collection procedure.

Musculoskeletal End Points. Aspects of bone quality and growth were assessed using a high-resolution pQCT bone scanner (XtremeCT, XtremeCT system II; Scanco Medical), digital radiography (tibia and femur lengths; Dragon Digital X-ray System using VXvue; Vieworks), and morphometric measurements (body and tail length). XtremeCT, radiography, and morphometry were performed on all animals under anesthesia once prior to initiation of dosing and at weeks 4, 8, 13, and 26. Animals were given an intramuscular injection of glycopyrrolate, ketamine $\mathrm{HCl}$ injection (United States Pharmacopeia), and dexmedetomidine prior to scanning, which was performed under isoflurane anesthesia. Atipamezole was administered (intramuscularly) following scanning to aid in recovery from anesthesia.

Bone Quality Measured by XtremeCT. XtremeCT was used to measure the volume of the right distal ulna growth plate in vivo and to measure overall bone quality, using the distal metaphysis and diaphysis of the right radius as a representative bone and bone location. Scans were performed using an XtremeCT system II (Scanco Medical). A volume of interest (VOI) was defined at the physis/ metaphysis and at the diaphysis, contoured, and analyzed. The reference line was placed at the distal aspect of the ulna, proximally to the styloid process, and included the widest part of the distal radius. For the radius metaphysis, the VOI was defined as all 125 slices. The first slice to contour on the radius was placed at a distance equal to $1 \%$ of the ulna length from the widest part of the distal radius and proximal to the growth plate. For the radius diaphysis, the VOI was defined as all 186 slices and was delimited using manual contouring of the first two-dimensional slice, then followed by automatic contouring using the "Iterate forward" function on all slices. For the ulna growth plate, the VOI was defined as all contoured slices. Contouring was performed as follows: for the total region, with an outer threshold of 74 and an inner threshold of 200, manual contouring was done, with auto fit, on the larger part of the distal ulna, proximal to the growth plate. This contour was then applied to the larger part of the ulna, distal to the growth plate. Then automatic contouring using the "morph" function was done between the two parts. For the distal region, automatic contouring on the distal ulna, distal to the growth plate, and

TABLE 3

In vitro NEP resistance, activity, and NPR-C affinity of CNP-38 conjugates

\begin{tabular}{|c|c|c|c|}
\hline CNP-38 Conjugate & NEP Resistance & Activity $^{a}$ & NPR-C Affinity ${ }^{b, c}$ \\
\hline (Linker Type, Attachment Site) & Half-Life $[\mathrm{h}]$ & [\%] & [\%] \\
\hline CNP-38 & $12.6 \pm 3.9^{d}$ & 100 & 100 \\
\hline 5-kDa PEG-linker CNP-38 (permanent, N-terminus) & Stable ${ }^{e}$ & 14 & $199 \pm 12$ \\
\hline 5-kDa PEG-linker CNP-38 (permanent, Lys26) & 65.4 & $<1^{f}$ & $83 \pm 7$ \\
\hline $2 \times 20$-kDa PEG-linker CNP-38 ${ }^{g}$ (transient, Lys 26 ) & $\mathrm{ND}^{h}$ & $<1^{f}$ & $11 \pm 4$ \\
\hline $4 \times 10-\mathrm{kDa}$ PEG-linker CNP-38 ${ }^{i}$ (permanent, Lys26) & Stable ${ }^{e}$ & $<1^{f}$ & $<2^{f}$ \\
\hline $4 \times 10$-kDa PEG-linker CNP-38 ${ }^{i}$ (transient, Lys26), TransCon CNP & ND & $<1^{f}$ & $<1^{f, j}$ \\
\hline $\mathrm{C}(10-\mathrm{kDa} \text { PEG-linker CNP-38 })_{4}{ }^{k}$ (permanent, Lys26) & ND & ND & $68 \pm 11$ \\
\hline
\end{tabular}

${ }^{a}$ Activity to elicit intracellular cGMP response in a NIH3T3 cell assay, relative to CNP-38.

${ }_{n} n=2$ to 3 .

${ }^{c}{\text { Ratio } \mathrm{IC}_{50} \text { CNP-38/IC }}_{50}$ CNP-38 conjugate.

$d_{n}=4$.

${ }^{e}$ Almost no degradation after $96 \mathrm{~h}$.

$f_{\text {Limitation due to assay range. }}$

${ }^{g}$ Branched $40-\mathrm{kDa}$ PEG carrier having the CNP attachment site near the branching site of two 20-kDa arms.

${ }^{h} \mathrm{ND}$, not determined.

${ }^{i}$ Branched 40-kDa PEG carrier having the CNP attachment site near the branching site of four 10-kDa arms.

${ }^{j}$ Calculated from a $25 \times$ concentrated sample.

${ }^{k}$ Branched $4 \times 10$-kDa PEG carrier having a total of four CNPs attached. The CNP attachment site is not close to the branching site of the four $10-\mathrm{kDa}$ arms, but at the distal end of each of the arms. 
for the proximal region, automatic contouring on the distal ulna, proximal to the growth plate, was applied. The evaluation method used is presented in Table 2, employing a three-dimensional constrained Gaussian filter with finite filter support (1 voxel) and filter width $(\sigma=0.8)$. The images were subsequently binarized to separate the object from background using a global thresholding procedure.

Bone Length Measurements. For the physical measurements of body length, the animal was placed on its back with the head straight and hindlimbs extended (with toes up), and measurements were done using a measuring tape from the top of the head to the heels. The tail length was measured from the base of the tail ventrally to the tip of the tail. Measurements were recorded in centimeters with one decimal place.

Digital radiographs of the right femur/tibia and the right radius/ ulna were obtained in ventro-dorsal view (one view for both sides, including the pelvis). Furthermore, medio-lateral views were taken of the right femur/tibia, and a cranio-caudal view was taken for the right radius/ulna. Radiographs were evaluated qualitatively and consisted of an evaluation of the bone, including assessment of the growth plate. Physeal closure (right proximal tibia and distal femur) was scored, as follows: 0 , widely open physis; 1 , open physis; 2 , physis partially closed; 3 , physeal scar; 4 , physis closed. Tibia and ulna lengths were derived from the digital radiographs using the following landmarks: Ulna, olecranon to styloid process and tibia, most proximal ossification center to the most distal ossification center.

Growth parameters were compared across groups using an analysis of covariance model of the relative change from baseline, including treatment as a fixed effect and the baseline value as a covariate. Group estimates and contrasts from this model are presented with $95 \%$ confidence intervals.

Histomorphometry. Growth plate histology was evaluated in the proximal tibial growth plate obtained from control and treated animals at terminal sacrifice. The epiphyseal plate width of the proliferative and hypertrophic zones was derived from tibial H\&E sections from controls and treated animals sacrificed following 26 weeks of treatment. One coronal H\&E section of $4 \mu \mathrm{m}$ in thickness and approximately $2 \mathrm{~cm}$ in length was taken from the proximal tibia at the level of the medial collateral ligament to include the articular cartilage, epiphysis, growth plate, metaphysis, and diaphysis. The proliferative zone was defined as the region of chondrocytes displaying a flattened-disc morphology and extending to the level where the cell assumed a more rounded cellular morphology. The hypertrophic zone was defined as the region beginning with the consistent cylindrical cellular profile and extending to the metaphyseal chondro-osseous junction. The width of the hypertrophic zone was calculated using an Image-Pro Plus image analysis system (MediaCybernetics).

\section{Results}

\section{In Vitro Screening of CNP Prodrugs: NEP Resistance, Activity, and NPR-C Affinity}

It was proposed by Oefner et al. (2000) that substrates of the NEP protease (like CNP) have a size limit of $<3 \mathrm{kDa}$ due to the restricted volume of the active site of the enzyme. Wendt et al.
(2015) confirmed short-term in vitro NEP resistance (140-minute exposure) of several CNP variants exceeding the $3-\mathrm{kDa}$ range, e.g., CNP-38 (4.1-kDa) or N-terminally PEGylated 2-kDa PEG CNP-22 (4.2-kDa). In our in vitro NEP resistance studies conducted over several days, CNP-38 was degraded slowly with a half-life of approximately 13 hours (Table 3). To further improve NEP resistance, a 5-kDa PEG was attached to the N-terminus of CNP-38, which resulted in almost complete resistance against NEP proteolysis (Table 3). In contrast, a 5-kDa PEG moiety attached to the Lys26 side chain of the ring part of CNP rendered the CNP molecule less resistant to proteolysis, resulting in a half-life of 65.4 hours. Initial NEP proteolysis was reported at the Cys6-Phe7 bond of CNP-22 (Watanabe et al., 1997). This is not supported by our findings, as the corresponding Cys22-Phe23 bond of CNP-38 should be protected more efficiently by the PEG attachment at the nearby Lys26 side chain, compared with the N-terminus (Leu1). Increasing the PEG size from a 5-kDa moiety to a larger 40-kDa branched PEG $(4 \times 10-\mathrm{kDa}$ PEG $)$ led to full NEP resistance of the Lys26 side chain conjugated CNP-38.

The biologic activity of the various CNP conjugates was further evaluated in vitro in a cell-based assay. Conjugation of the 5-kDa PEG at the N-terminus of CNP-38 did not fully prevent NPR-B binding and resulted in $14 \%$ residual potency. In contrast, conjugation of the 5-kDa PEG to the Lys26 side chain of CNP-38 effectively reduced potency to below $1 \%$ (Table 3). This is not surprising as Lys26 is part of the Leu-Lys-Leu motif of the CNP intramolecular ring structure that was reported by Furuya et al. (1992) as essential for CNP binding to NPR-B and subsequent activation of the cGMP signaling pathway. For the $4 \times 10$-kDa PEG Lys26 side chain conjugate (TransCon CNP), a low activity of $<1 \%$ was also evident.

The affinity of the CNP-38 PEG conjugates to the NPR-C receptor was screened for in a competitive ligand-binding cell assay. PEG 5-kDa conjugation to the $\mathrm{N}$-terminus or Lys26 side chain of CNP-38 did not lead to reduced affinity to NPR-C compared with CNP-38. He et al. (2005) have shown that the NPR-C receptor interacts with the intramolecular ring structure of CNP-22. The four N-terminal amino acids of CNP-22 are largely disordered and are pointing away from the binding cleft and hence do not impact binding to NPR-C. Consequently, further screening focused on PEG conjugates of the Lys26 side chain of CNP-38. Using larger, for example, 40-kDa PEG conjugates, reduced affinities of the CNP-38 PEG conjugates were obtained and were furthermore strongly influenced by the architecture of the PEG molecule. An affinity of $<1 \%$ was obtained for a branched $40-k D a$ PEG conjugate with the CNP attachment site close to the branching site of the four $10-\mathrm{kDa}$ arms $(4 \times 10-\mathrm{kDa}$ PEG) (Table 3$)$. In contrast, a $4 \times$ $10-\mathrm{kDa}$ PEG conjugate with CNP-38 attached away from the

TABLE 4

Mean pharmacokinetic parameters of CNP (after first dose) released from TransCon CNP following administration of TransCon CNP compared with the daily CNP-39 molecule in monkeys

\begin{tabular}{lcccc}
\hline \multicolumn{1}{c}{ Group } & Dose $(\mu \mathrm{g} \mathrm{CNP} / \mathrm{kg})$ & $\mathrm{T}_{\max }{ }^{a}(\mathrm{~h})$ & $C_{\max }(\mathrm{pmol} / /)$ & $\mathrm{AUC}_{(0-\mathrm{t})}(\mathrm{h} \times \mathrm{pmol} / \mathrm{l})$ \\
\hline Daily CNP-39 molecule & $20 / \mathrm{day}$ & 0.25 & 3850 & 2560 \\
TransCon CNP & $40 / \mathrm{wk}$ & 48 & 16.5 & 1748 \\
TransCon CNP & $100 / \mathrm{wk}$ & 24 & 39.4 & 4186 \\
\hline
\end{tabular}

AUC, area under the curve; $\mathrm{T}_{1 / 2}$, half-life; $\mathrm{T}_{\max }$, time to maximum plasma concentration.

${ }^{a}$ Median $\mathrm{T}_{\max }$ is presented. 


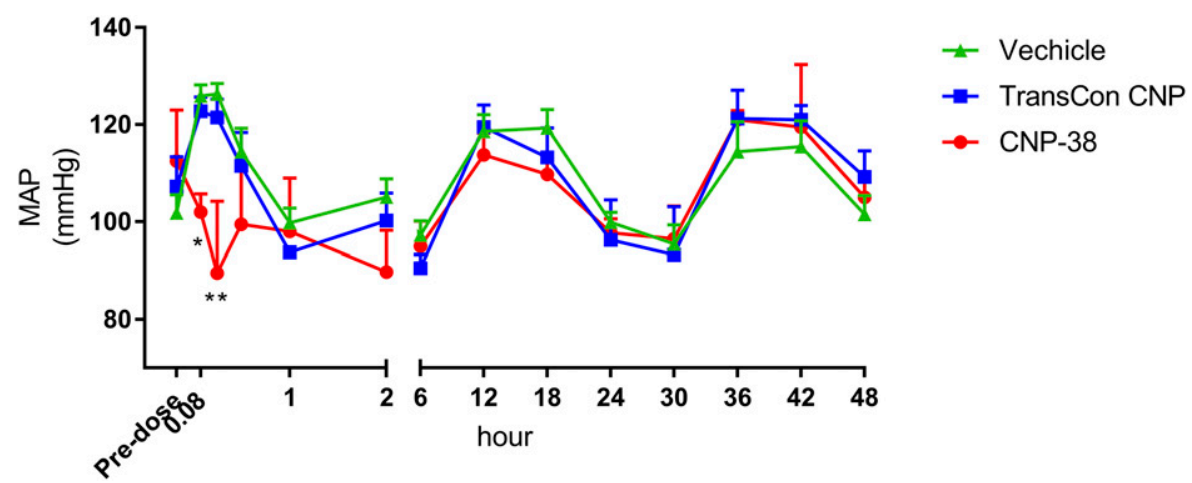

Fig. 1. MAP following a single subcutaneous administration of either vehicle, CNP-38 $(800 \mu \mathrm{g}$ $\mathrm{CNP} / \mathrm{kg})$, or TransCon CNP (800 $\mu \mathrm{g} \mathrm{CNP} / \mathrm{kg})$ to mice. Footnote: MAP recorded up to 48 hours postdose. Means ( \pm S.E.M.). *Significant difference from vehicle $P<0.05$. **Significant difference from vehicle $P<0.01$. William's test comparing treatment groups with vehicle control at each time point. branching site, at the distal ends of each of the $10-\mathrm{kDa}$ arms $\left[\mathrm{C}\left(10-\mathrm{kDa}\right.\right.$ PEG CNP-38) $\left.{ }_{4}\right]$, did not significantly reduce affinity to NPR-C. Keeping the attachment site close to the branching point, but decreasing the number of arms from four to two $(2 \times 20$-kDa PEG), had less effect on the NPR-C receptor affinity compared with the $4 \times 10-\mathrm{kDa}$ PEG conjugate.

\section{Pharmacokinetics of TransCon CNP and a Daily CNP-39 Molecule in Cynomolgus Monkey}

The daily CNP-39 molecule was rapidly absorbed into the systemic circulation following subcutaneous administration to cynomolgus monkeys with a time to maximum plasma concentration ranging between 5 minutes and 1 hour postdose. $C_{\max }$ was followed by a rapid decline with an associated half-life of approximately 21 minutes. The last measurable time point was 2 hours postdose (LLOQ $37 \mathrm{pmol} / \mathrm{l})$. Based on the half-life, only approximately $1.5 \%$ of $C_{\max }$ was estimated to be still in the systemic circulation at 4 hours postdose. Following repeated daily administration of the daily CNP-39 molecule, an apparent increase in both $C_{\max }$ and half-life was observed during the time course of the study: from the first dose administration till the last administration in week 26 . Following administration of TransCon CNP, released CNP-38 was measured in the systemic circulation following the 1st and 26th dose. On both dosing occasions, CNP-38 was quantifiable for up to 168 hours postdose at both 40 and $100 \mu \mathrm{g}$ CNP/kg. The main PK parameters from the first dose administration are presented in Table 4.

As indicated above, CNP-38 released from TransCon CNP has a half-life of about 90 hours, indicating that sustained increased systemic CNP levels are achieved in cynomolgus monkey following weekly dosing with TransCon CNP. The systemic peak levels of CNP-38 following administration of TransCon CNP (100 $\mu \mathrm{g} / \mathrm{kg})$ were estimated to be almost 100fold lower than the $C_{\max }$ for the daily CNP-39 molecule

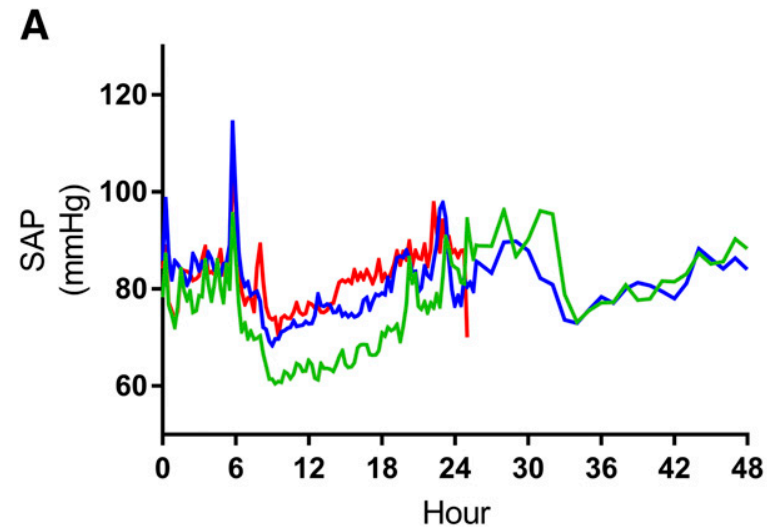

B

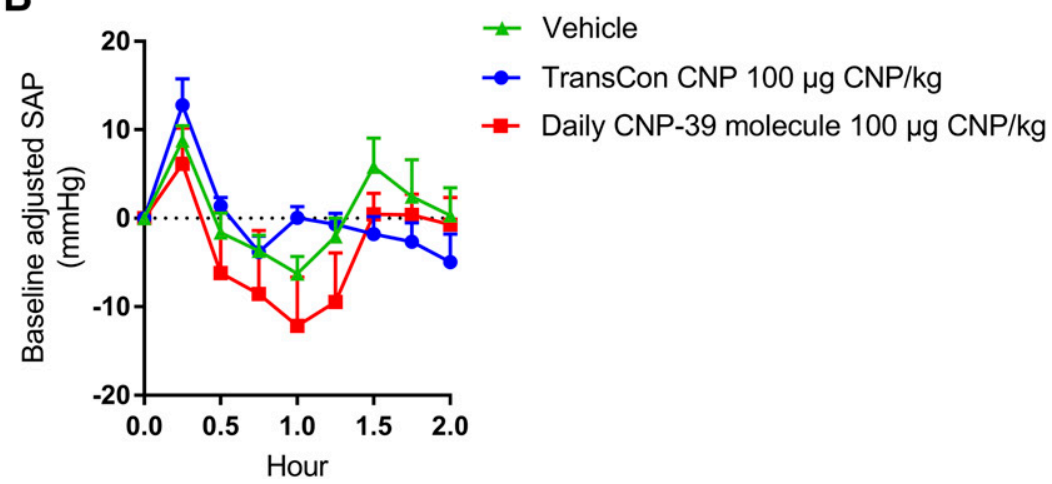

Fig. 2. SAP following a single subcutaneous administration of TransCon CNP and daily CNP-39 molecule to cynomolgus monkeys compared with vehicle control. Footnote: SAP recorded up to 48 hours postdose. (A) Means. (B) Baseline-adjusted means ( \pm S.E.M.). 
TABLE 5

Bone length [millimeters [95\% confidence interval] (\% change from control)] determined by X-ray after 5 wk of daily bolus injection or continuous infusion of CNP-38 or vehicle control in FVB mice

\begin{tabular}{lcccc}
\hline & \multicolumn{2}{c}{ Subcutaneous Bolus Injection } & \multicolumn{2}{c}{ Subcutaneous Continuous Infusion } \\
\hline Measurement & Vehicle Control & CNP-38 203 $\mu \mathrm{g} / \mathrm{kg}$ Per Day & Vehicle Control & CNP-38 203 $\mu \mathrm{g} / \mathrm{kg}$ Per Day \\
Femur length $^{a}$ & $14.2[13.7 ; 14.8]$ & $15.0[14.5 ; 15.6](5.5 \%)^{*}$ & $13.6[13.0 ; 14.1]$ & $14.6[14.1 ; 15.2](7.1 \%)^{*}$ \\
Tibia length $^{b}$ & $17.9[17.7 ; 18.1]$ & $18.7[18.5 ; 18.9](4.0 \%)^{*}$ & $17.6[17.4 ; 17.8]$ & $19.8[19.6 ; 20.0](12.2 \%)^{*}, \#$ \\
Spine length $^{c}$ & $53.8[52.4 ; 55.2]$ & $59.8[58.5 ; 61.2](11.3 \%)^{*}$ & $53.6[52.2 ; 55.0]$ & $67.0[65.6 ; 68.4](25.0 \%)^{*}, \#$ \\
\hline${ }^{a}$ Right femur. & & \\
${ }^{b}$ Right tibia. & & & \\
${ }^{c}$ Lateral view. & & &
\end{tabular}

(20 $\mu \mathrm{g} / \mathrm{kg}$ ). In contrast to the daily CNP-39 molecule, which was cleared rapidly from the systemic circulation, resulting in a dose holiday of close to 20 hours, the systemic CNP-38 level following subcutaneous TransCon CNP administration was above the LLOQ of the assay ( $2 \mathrm{pmol} / \mathrm{l})$ throughout the 168-hour sampling period.

Cardiovascular Assessment in Mice. Dose-dependent reductions in $\mathrm{BP}$ were observed in mice administered a s.c. dose of $800 \mu \mathrm{g} / \mathrm{kg}$ unconjugated CNP-38. The lowest MAP was recorded at 2 hours postdose and subsided at about 12 hours postdose, and no effect on HR was observed (Fig. 1). No TransCon CNP-related effects on BP or HR were recorded at a dose level of $800 \mu \mathrm{g} \mathrm{CNP} / \mathrm{kg}$ administered as the TransCon CNP prodrug (Fig. 1).

Cardiovascular Assessment in Cynomolgus Monkeys. In cynomolgus monkeys, a single s.c. administration of TransCon CNP at 10,40 , and $100 \mu \mathrm{g} \mathrm{CNP} / \mathrm{kg}$ or the daily CNP-39 molecule at $50 \mu \mathrm{g} \mathrm{CNP} / \mathrm{kg}$ resulted in no changes in systemic BP, HR, ECG parameters, or body temperature up to 48 or 24 hours postdose, respectively. Three of the four male monkeys were successfully administered the daily CNP-39 molecule at $100 \mu \mathrm{g} \mathrm{CNP} / \mathrm{kg}$, whereas one animal received only $60 \mu \mathrm{g} \mathrm{CNP} / \mathrm{kg}$. The remaining three animals that received the full dose of CNP-39 had decreased SAP between 0.25 and 1.25 hours postdose (from $19 \%$ to $28 \%$ compared with predose). Exposure measured as total prodrug (data not shown) was confirmed in all animals administered TransCon CNP at 48 and 168 hours postdose, with the exception of two animals administered $40 \mu \mathrm{g} \mathrm{CNP} / \mathrm{kg}$ for which the exposure, for unknown reasons, could not be confirmed. In the remaining animals, the plasma concentrations of the TransCon CNP prodrug were similar across animals with respect to the dose levels and time points. The effect on SAP is presented below (Fig. 2).

Stimulation of Bone Growth by Daily Subcutaneous Injection or Continuous Infusion of CNP-38 in Mice. Three-week-old male FVB mice $(n=9)$ received CNP-38 at a dose of $203 \mu \mathrm{g} / \mathrm{kg}$ per day either as a daily subcutaneous bolus injection or as continuous subcutaneous infusion by osmotic Alzet pump. CNP-38 administered via both routes were generally well tolerated, and animal body weights were developed according to historical FVB mouse growth charts (data not shown). Weekly measurements showed a sustained growth measured as animal body length during the entire study period, following both daily subcutaneous bolus injection and continuous subcutaneous infusion (data not shown). CNP-38 given as a daily bolus elicited significant growth in the appendicular and axial skeleton (Table 5).

Applying the same amount of CNP-38 per day as a continuous subcutaneous infusion led to an even more pronounced growth effect, and significant increases in responses on several growth parameters were observed as compared with the daily subcutaneous bolus injection (Table 5).

\section{Efficacy in Monkey}

Biomarkers of Bone Turnover. Over the 26-week study period, control animals generally presented slight increases in bone formation markers associated with stable values or slight decreases in bone resorption markers, indicating bone turnover in favor of bone formation (data not shown). During the treatment period, TransCon CNP at $40 \mu \mathrm{g}$ CNP/kg per week did not show any meaningful changes in serum BAP, PINP, or OC levels. Animals administered TransCon CNP at $100 \mu \mathrm{g} \mathrm{CNP} / \mathrm{kg}$ per week or the daily CNP-39 molecule at $20 \mu \mathrm{g} \mathrm{CNP} / \mathrm{kg}$ per day, in contrast, presented increases in serum BAP and PINP levels at week 26 as compared with controls (Table 6). No meaningful impact of TransCon CNP or treatment with the daily CNP-39 molecule was observed on serum OC and serum CTX levels or urine NTx levels (data not shown).

Bone Growth. During the treatment period, there were no meaningful treatment-related changes in growth plate closure at the right femur, tibia, and radius/ulna (proximal and distal physis).

TABLE 6

Percent increase in bone formation markers BAP and PINP following weekly TransCon CNP or daily CNP-39 molecule administration for 26 wk to cynomolgus monkeys

\begin{tabular}{lccc}
\hline & $\begin{array}{c}\text { TransCon CNP 40 } \mu \mathrm{g} \mathrm{CNP} / \mathrm{kg} \text { Per } \\
\text { Week }\end{array}$ & $\begin{array}{c}\text { TransCon CNP 100 } \mu \mathrm{g} \text { CNP/kg Per } \\
\text { Week }\end{array}$ & $\begin{array}{c}\text { Daily CNP-39 Molecule 20 } \mu \mathrm{g} / \mathrm{kg} \text { Per } \\
\text { Day }\end{array}$ \\
\hline $\begin{array}{l}\text { No. animals per } \\
\text { group }\end{array}$ & 4 & 4 & 4 \\
BAP & - & $14^{a}$ & $51^{a}$ \\
PINP & - & $53^{a}$ & $144^{a}$ \\
\hline
\end{tabular}

${ }^{a}$ Significantly different from control group; $P<0.05$, based on statistical analysis of group means. 
TABLE 7

Estimated relative effects of CNP treatment on growth of body, tail, tibia, and ulna (relative increase in growth compared with vehicle treatment [\% (95\% confidence interval) in monkeys]

\begin{tabular}{lccc}
\hline & TransCon CNP $40 \mu \mathrm{g}$ CNP/kg Per Week & TransCon CNP $100 \mu \mathrm{g}$ CNP/kg Per Week & Daily CNP-39 Molecule 20 $\mu$ g CNP/kg Per Day \\
\hline Body & $1.0(-2.3 ; 4.4)$ & $4.8(1.1 ; 8.5)^{*}$ & $3.3(0.0 ; 6.6)^{*}$ \\
Tail & $1.4(-2.7 ; 5.5)$ & $8.6(3.7 ; 13.4)^{*}$ & $2.8(-1.3 ; 6.9)$ \\
Tibia & $1.7(-2.0 ; 5.4)$ & $6.0(2.1 ; 9.9)^{*}$ & $2.9(-1.0 ; 6.8)$ \\
Ulna & $0.9(-2.8 ; 4.7)$ & $2.7(-1.1 ; 6.5)$ & $0.8(-2.9 ; 4.5)$ \\
\hline
\end{tabular}

${ }^{*} P<0.05$.

Compared with the observed growth in control animals, body length was increased by $5 \%$ and $3 \%$, and tail length by 9\% and 3\%, at the end of treatment, for TransCon CNP and the daily CNP molecule, respectively (Table 7).

A linear growth over the 26-week treatment period was evident for both tibia and ulna (Fig. 3A) for all dose groups, as expected for growing juvenile monkeys. Weekly dosing of TransCon CNP to the skeletally immature cynomolgus monkey at a dose of $40 \mu \mathrm{g} \mathrm{CNP/kg} \mathrm{per} \mathrm{week} \mathrm{generally} \mathrm{resulted} \mathrm{in}$ higher values for tibia length (most notably at week 26), as well as in body and tail lengths compared with controls. At $100 \mu \mathrm{g} \mathrm{CNP/kg} \mathrm{per} \mathrm{week,} \mathrm{significant} \mathrm{increases} \mathrm{in} \mathrm{tibia} \mathrm{length}$ were observed (Fig. 3B), amounting to a $6 \%$ increased length of tibia at week 26 compared with control animals (Table 7). Administration of the daily CNP-39 molecule resulted in an increase in tibia length of $3 \%$ at week 26 compared with control.

Animals treated at $100 \mu \mathrm{g} \mathrm{CNP} / \mathrm{kg}$ per week also exhibited an increase in right ulna length of $3 \%$, whereas no meaningful changes in ulna length were observed in animals administered the daily CNP-39 molecule or $40 \mu \mathrm{g}$ CNP/kg per week group as compared with control animals.

Bone Quality (XtremeCT). In TransCon CNP and daily CNP-39 molecule-treated animals, no meaningful changes in the bone quality parameters were observed. Likewise, the distal ulna growth plate volume was not impacted by weekly TransCon CNP or daily CNP-39 molecule treatment (data not shown). At the distal radius metaphysis, controls presented signs of bone consolidation as expected for a growing animal evidenced by slight increases in the apparent density of the total region (vBMD) as assessed by XtremeCT (data not shown). In the trabecular bone compartment, there were slight increases in the overall bone volume fraction and in vBMD, characterized by slight increases in trabecular number, thickness, and separation (Table 8).

In the cortical compartment of the distal metaphysis of the radius, slight increases in cortical thickness were associated with slight decreases in tissue bone mineral density and vBMD (Table 8).

In the distal radius diaphysis (Table 8), controls presented signs of bone growth as evidenced by slight increases in cortical thickness associated with marginal decreases in bone mineral density (BMD) and slight decreases in vBMD. Other parameters investigated remained stable over time. Controls presented slight increases in the distal ulna growth plate volume over time (data not shown).

Histomorphometry. TransCon CNP at $100 \mu \mathrm{g} \mathrm{CNP/kg}$ per week and the daily administered CNP analog at $20 \mu \mathrm{g}$ $\mathrm{CNP} / \mathrm{kg}$ per day induced statistically significant higher mean proliferative or hypertrophic zone widths of the proximal tibial physis compared with control animals (Table 9). In comparison with control animals, the width of the proliferative zone for TransCon CNP was increased by $37 \%$ compared with $16 \%$ for the daily CNP-39 molecule. The width of the hypertrophic zones was similar for the weekly TransCon CNP and the daily CNP39 molecule and amounted to an increase in width of $38 \%$ to $39 \%$ compared with control animals. These higher mean proliferative and hypertrophic zone widths correlated microscopically with an increased cellularity of the proliferative and hypertrophic zones, respectively, and a statistically significant increase of $14 \%$ in the thickness of the growth plate for TransCon CNP-treated animals (Fig. 4). There were no statistically significant histomorphometry changes in the proximal tibial physis of monkeys administered TransCon CNP at $40 \mu \mathrm{g}$ $\mathrm{CNP} / \mathrm{kg}$ per week.

\section{Safety}

During the 26-week treatment period, no abnormal clinical signs were observed for any of the treatment regimens, including observations before and after $C_{\max }$ for both the weekly and the daily CNP-39 molecule. No changes in standard clinical chemistry and hematology were observed, and no impact on serum A-type natriuretic peptide levels was observed. Furthermore, histopathological assessment by H\&E (Society of Toxicologic Pathology recommended organ list) did not suggest any adverse effects of CNP.

\section{Discussion}

Based on the presented in vitro screenings, the CNP-38 PEG $4 \times 10-\mathrm{kDa}$ conjugate TransCon CNP was selected for clinical development. This prodrug of CNP-38 exhibited an almost complete resistance against NEP proteolysis and very low NPR-C affinity and low ability to activate NPR-B. The interaction with NPR-B and NPR-C was markedly reduced by attaching the PEG carrier to the Lys26 side chain of CNP-38, which is part of the intramolecular ring structure that interacts with the active binding site for both receptors (Igaki et al., 1998). A 5-kDa PEG attached to CNP-38 was found sufficient to reduce binding to and activation of NPR-B, whereas a four-arm PEG $4 \times 10-\mathrm{kDa}$ moiety with the CNP attachment close to the PEG branching site was required to additionally minimize NPR-C binding. This is in agreement with the crystal structure of the NPR-C CNP complex, which shows only weak interaction between CNP's Lys26 side chain, but stronger interactions between other hydrophobic motifs of the peptide and the receptor (He et al., 2005), which also binds A- and B-type natriuretic peptides. NEP resistance could be achieved through attachment of a $40-\mathrm{kDa}$ PEG carrier to the Lys26 side chain (and may also be beneficial for the protection against potential other proteolytic pathways). 
A

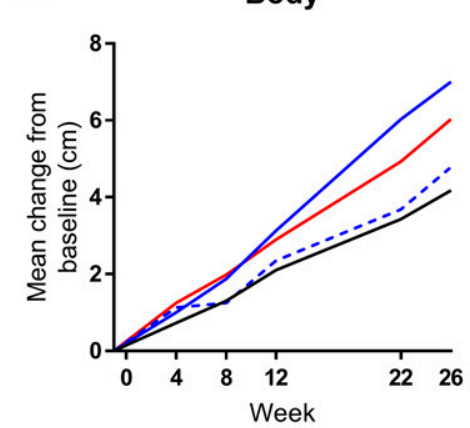

Tibia

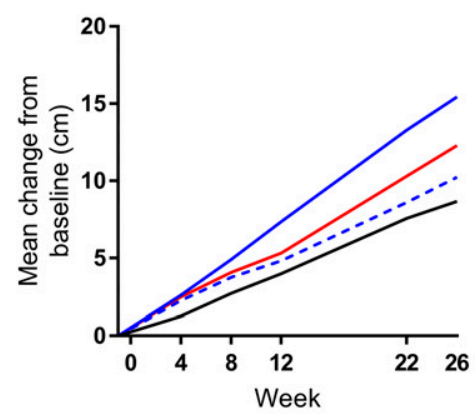

Tail

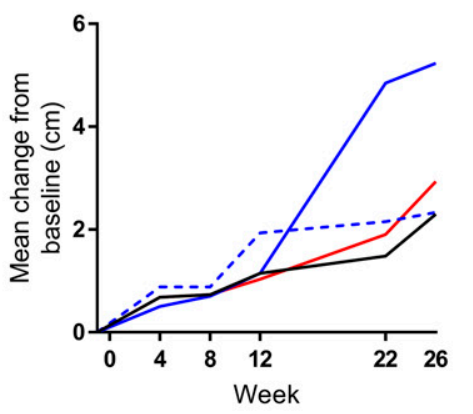

Ulna

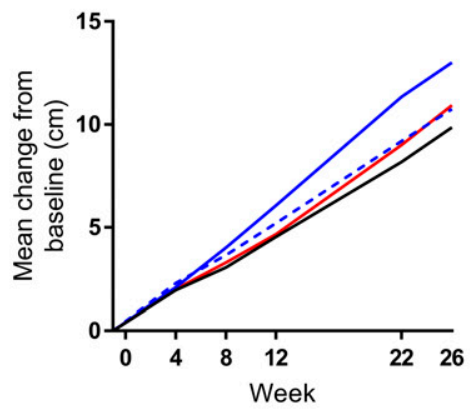

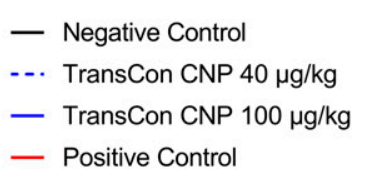

B

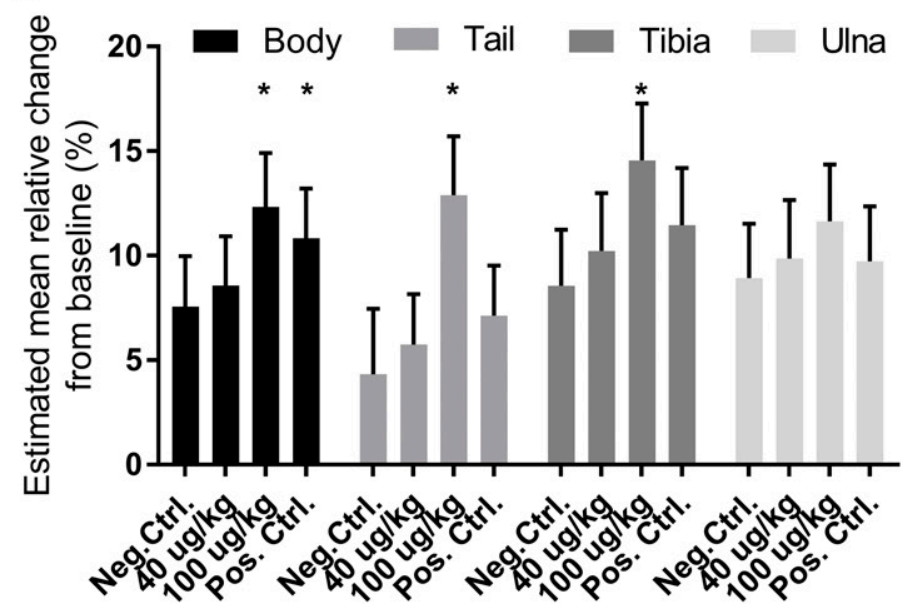

Fig. 3. Growth from baseline of body, tail, tibia, and ulna following weekly administration of TransCon CNP to monkeys ( $n=4 /$ group) compared with the daily CNP-39 molecule (positive control) and vehicle treatment (negative control); (A) for duration of study (26 weeks), and (B) comparing relative change from baseline $( \pm 95 \%$ CI $)$ at week 26 . Footnote: Estimates and test are derived from an analysis of covariance model, including treatment as a fixed effect and baseline value as a covariate. *Significant difference in absolute growth from negative control, $P<0.05$. Neg.Ctrl, negative control; Pos.Ctrl, positive control.

The extent of the growth of tibia, femur, and spine in the FVB mice after bolus injection of CNP-38 at $203 \mu \mathrm{g} / \mathrm{kg}$ per day $(50 \mathrm{nmol} / \mathrm{kg}$ per day) is comparable to the results reported for $287 \mu \mathrm{g} / \mathrm{kg}$ per day (70 $\mathrm{nmol} / \mathrm{kg}$ per day) bolus injection of vosoritide, employing a similar experimental design (Wendt et al., 2015). Overall growth effects on femur, tibia, and spine were much stronger when CNP-38 was applied as a continuous subcutaneous infusion compared with a daily subcutaneous bolus injection indicating improved efficacy as a result of continuous exposure. For tibia and spine, the continuous infusion of CNP more than doubled the effect, compared with daily bolus administration and hence intermittent exposure of CNP-38, suggesting that bone growth, even in a healthy animal with normal endogenous CNP levels, could be greatly accelerated by sustained systemic or local CNP exposure. Yasoda et al. (2009) tested the impact on bone growth of continuous infusion of CNP-22 in 3-week-old mice. Only intravenous infusion of high doses of CNP-22 $(1 \mu \mathrm{g} / \mathrm{kg}$ per minute $=655 \mathrm{nmol} / \mathrm{kg}$ per day $)$ led to significant effects on bone growth, whereas subcutaneous injections of similar doses of CNP-22 did not produce significant effects. This discrepancy between the effect of subcutaneous dosing of CNP-38 and CNP-22 can be explained by the relatively fast inactivation of the CNP-22 
TABLE 8

Summary of XtremeCT values (mean \pm S.D.) as percentage change from acclimation period $(n=4)$ after weekly subcutaneous administration of TransCon CNP or daily CNP-39 molecule for $26 \mathrm{wk}$

\begin{tabular}{|c|c|c|c|c|c|}
\hline \multirow{2}{*}{$\begin{array}{l}\text { Treatment } \\
\text { CNP Dose }\end{array}$} & & \multirow{2}{*}{$\begin{array}{c}\text { Control } \\
\text { Vehicle }\end{array}$} & \multirow{2}{*}{$\begin{array}{c}\text { Daily CNP-39 Molecule } \\
20 \mu \mathrm{g} / \mathrm{kg} \text { Per Day }\end{array}$} & \multicolumn{2}{|c|}{ TransCon CNP } \\
\hline & & & & $40 \mu \mathrm{g} / \mathrm{kg}$ Per Week & $100 \mu \mathrm{g} / \mathrm{kg}$ Per Week \\
\hline & \multicolumn{5}{|c|}{ Right Radius, Distal Metaphysis-Trabecular Region } \\
\hline Trabecular region vBMD & $\mathrm{mg} \mathrm{HA} / \mathrm{cm} 3$ & $19.8 \pm 10.3$ & $18.2 \pm 14.9$ & $27.0 \pm 17.9$ & $16.5 \pm 10.7$ \\
\hline Trabecular number & $1 / \mathrm{mm}$ & $13.2 \pm 11.6$ & $5.8 \pm 6.0$ & $12.9 \pm 8.8$ & $8.9 \pm 5.3$ \\
\hline Trabecular thickness & $\mathrm{mm}$ & $4.6 \pm 3.7$ & $8.9 \pm 6.7$ & $16.2 \pm 14.5$ & $14.0 \pm 11.8$ \\
\hline Trabecular separation & $\mathrm{mm}$ & $-14.6 \pm 8.4$ & $-5.9 \pm 7.0$ & $-12.0 \pm 9.0$ & $-10.5 \pm 5.0$ \\
\hline Cortical tissue BMD & $\mathrm{mg} \mathrm{HA} / \mathrm{cm} 3$ & $-3.8 \pm 1.4$ & $-5.9 \pm 1.6$ & $-5.9 \pm 1.2$ & $-2.4 \pm 1.8$ \\
\hline Cortical thickness & $\mathrm{mm}$ & $-3.6 \pm 16.0$ & $-9.4 \pm 11.2$ & $-16.7 \pm 11.5$ & $4.5 \pm 10.7$ \\
\hline \multicolumn{6}{|c|}{ Right Radius, Distal Diaphysis-Cortical Region } \\
\hline Cortical region vBMD & $\mathrm{mg} \mathrm{HA} / \mathrm{cm} 3$ & $-1.8 \pm 1.6$ & $-3.3 \pm 1.6$ & $-7.8 \pm 9.8$ & $-2.4 \pm 2.3$ \\
\hline Cortical tissue BMD & $\mathrm{mg} \mathrm{HA} / \mathrm{cm} 3$ & $-4.8 \pm 1.9$ & $-6.7 \pm 1.4$ & $-6.3 \pm 3.6$ & $-3.7 \pm 1.6$ \\
\hline Cortical thickness & $\mathrm{Mm}$ & $7.0 \pm 3.0$ & $8.2 \pm 5.0$ & $1.1 \pm 8.4$ & $8.9 \pm 4.7$ \\
\hline
\end{tabular}

BMD, bone mineral density; BV, bone volume; TV, tissue volume.

by NEP in mice compared with the increased stability of CNP-38 toward NEP shown in vitro.

Also, in healthy juvenile monkeys, TransCon CNP dosed subcutaneous once weekly gave rise to significant increases in growth based on several morphometric outcomes [body, tail, and long bones (tibia and ulna)]. The effect on ulna was only observed for TransCon CNP and varied markedly between animals, which may explain why no significant change in ulna growth plate volume was observed in the current study by XtremeCT. Wendt et al. (2015) likewise reported lack of a significantly increased volume of the right proximal tibial growth plate, following treatment with vosoritide in the same dose range as applied in the current study, whereas tibial growth plate expansion was evident when measured at the histologic level. This further suggests that the different methodologies employed in assessing growth plate dynamics vary greatly in their ability to pick up growth-promoting effects at the growth plate level.

At $100 \mu \mathrm{g} / \mathrm{kg}$ per week TransCon CNP, the observed relative growths were at least comparable to that observed for $20 \mu \mathrm{g} / \mathrm{kg}$ per day CNP-39 (observed ratios between relative growth outcomes were 1.4-3.3), and this despite the lower overall weekly dose of TransCon CNP (100 $\mu \mathrm{g} / \mathrm{kg}$ ) relative to the accumulated CNP dose following daily administration of $20 \mu \mathrm{g} / \mathrm{kg}$ CNP-39. Based on the histomorphometric analysis of the tibial growth plate, a more marked effect of TransCon $\mathrm{CNP}$ on the proliferating zone, giving rise to an overall greater and significant expansion of the growth plate thickness as such, does suggest that continuous exposure to CNP may afford a better effect on the growth plate and thereby on bone growth, relative to intermittent CNP exposure, hereby confirming the observations from the murine continuous subcutaneous infusion study described above. The associated increases in the bone formation markers BAP and/or PINP, most likely due to osteoblast mediated increase in the subphyseal bone observed in the proximal tibial bone following both TransCon CNP (100 $\mu \mathrm{g} \mathrm{CNP/kg} \mathrm{per} \mathrm{week)} \mathrm{and} \mathrm{daily} \mathrm{administration} \mathrm{of} \mathrm{the}$ daily CNP-39 molecule ( $20 \mu \mathrm{g} \mathrm{CNP} / \mathrm{kg}$ per day), suggest that BAP and/or P1NP could be used as noninvasive markers for bone growth mediated by a CNP molecule.

In comparison with endogenous CNP-22, which has a half-life of 2 to 3 minutes, CNP-38 released from TransCon CNP exhibits a half-life in monkeys and in humans (https:// ascendispharma.gcs-web.com/static-files/1dd5844f-1f4f-4086b6d1-eb6d4ca43985) of approximately 90 hours. This pronounced extension in half-life of this otherwise extremely labile peptide underlines the excellent features of the TransCon platform. The TransCon technology platform has been clinically validated across three different products: TransCon hGH, for treatment of growth hormone deficiency (Phase 3); TransCon PTH for hypoparathyroidism (Phase 1); and TransCon CNP for treatment of comorbidities associated with ACH (Phase 1).

As indicated above, TransCon CNP gives rise to close to 100fold lower $C_{\max }$ compared with the CNP-39 molecule. High concentrations of CNP cause vasodilation, and decreased BP in animals (Lopez et al., 1997; Wendt et al., 2015) and intravenous bolus administration of CNP-22 in humans induces postdose hypotension coinciding with the maximum blood

TABLE 9

Histomorphometry data of the proximal tibial growth plate following weekly administration of TransCon CNP compared with the daily CNP-39 molecule for 26 wk to monkeys (\% change from vehicle control)

Treatment

TransCon CNP $40 \mu \mathrm{g}$ CNP/kg Per Week TransCon CNP $100 \mu \mathrm{g}$ CNP/kg Per Week Daily CNP-39 Molecule $20 \mu \mathrm{g} / \mathrm{kg}$ Per Day

\begin{tabular}{lcrr}
\hline No. animals per group & $\mathbf{4}$ & $\mathbf{4}$ & $\mathbf{4}$ \\
Bone, tibia (no. measured) & 4 & 4 & 4 \\
Proliferative zone width & -2 & $37^{a}$ & $16^{a}$ \\
Hypertrophic zone width & 7 & $38^{a}$ & $39^{a}$ \\
Epiphyseal plate thickness & -4 & $16^{a}$ & 7 \\
\hline
\end{tabular}

\footnotetext{
${ }^{a}$ Significantly different from control group; $P \leq 0.05$, based on statistical analysis of group means.
} 
A

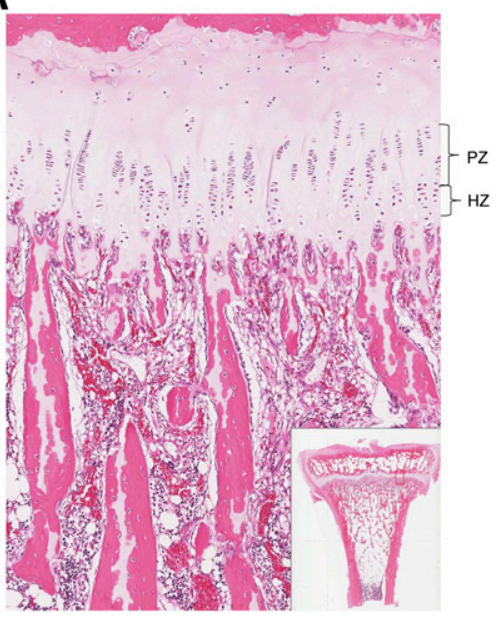

B

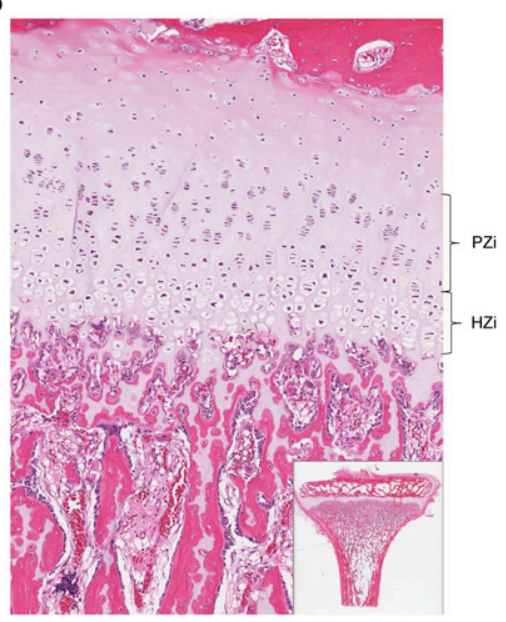

Fig. 4. Histomorphometry imaging of the proximal tibial physis following weekly administration of (A) vehicle control compared with (B) TransCon CNP (100 $\mu \mathrm{g}$ CNP/kg per week) for 26 weeks to monkeys. Footnote: PZ, proliferative zone; HZ, hypertrophic zone; PZi, increased cellularity and width of proliferative zone; HZi, increased cellularity and width of hypertrophic zone. concentrations $\left(C_{\max }\right)$, indicating a causal relationship (Igaki et al., 1998). The hypotensive effect of high systemic CNP concentrations is speculated to be mediated either via the normal paracrine effect of CNP on the vascular smooth muscle cells via NPR-C interaction (Lopez et al., 1997; Chauhan et al., 2003) or directly via NPR-B activation in endothelial cells, but could also result from competitive inhibition of, for example, A-type natriuretic peptide clearance via NPR-C (Igaki et al., 1998), or be a chronotropic effect on the sinoatrial node suggested to be mediated by NPR-B (Beaulieu et al., 1996, 1997).

By avoiding high peak CNP levels, a sustained-release CNP construct such as TransCon CNP holds great promise for reduced cardiovascular side effects compared with daily CNP bolus administration. Studies in mice confirmed that hemodynamic tolerability is greater when CNP-38 is conjugated to TransCon compared with unconjugated CNP-38. Furthermore, the hemodynamic effect caused by unconjugated CNP-38 and the daily CNP-39 molecule generally occurred within the first hour after dosing and did not occur in all animals. In agreement with previously reported data for CNP variants and the expected vasodilatory response of $\mathrm{CNP}$, administration of unconjugated CNP-38 and the daily CNP-39 molecule resulted in decreased BP (Clavell et al., 1993; Barr et al., 1996; Igaki et al., 1998; Scotland et al., 2005; Wendt et al., 2015). In contrast, no adverse cardiovascular effects were observed following single or repeated weekly doses of TransCon CNP in cynomolgus monkeys at $100 \mu \mathrm{g}$ CNP/kg. The good cardiovascular safety profile was confirmed in the first human clinical trial with TransCon CNP, in which single doses up to $150 \mu \mathrm{g} \mathrm{CNP} / \mathrm{kg}$ were administered to adult healthy volunteers without associated significant adverse cardiovascular effects (https://ascendispharma.gcs-web.com/ static-files/1dd5844f-1f4f-4086-b6d1-eb6d4ca43985).

Overall, TransCon CNP shows great promise in providing an efficacious and safe therapeutic modality in $\mathrm{ACH}$, by providing continuous exposure to promote bone growth, while avoiding high systemic CNP concentrations that can induce cardiovascular side effects.

\section{Acknowledgments}

We thank Bettina Lust, Franziska Gille, Nicole Hassepaß, Nora Kaluza, Steffen Schröder, and Tom Woods for the preparation of the CNP compounds; Bioassay, Heidelberg, for development and conduct of the activity and NPR-C affinity studies; and staff at Charles River
Laboratories, Montreal and Edinburgh, and at ENVIGO, Huntingdon, for conduct of the in vivo studies.

\section{Authorship Contributions}

Participated in research design: Breinholt, Rasmussen, Hersel, Zettler.

Conducted experiments: Breinholt, Rasmussen, Hersel, Bernhard, Faltinger.

Performed data analysis: Breinholt, Rasmussen, Mygind, Kjelgaard-Hansen, Hersel, Zettler, Faltinger, Bernhard.

Wrote or contributed to the writing of the manuscript: Breinholt, Kjelgaard-Hansen, Rasmussen, Mygind, Hersel, Zettler.

\section{References}

Abbey SE and Potter LR (2003) Lysophosphatidic acid inhibits C-type natriuretic peptide activation of guanylyl cyclase-B. Endocrinology 144:240-246.

Barr CS, Rhodes P, and Struthers AD (1996) C-type natriuretic peptide. Peptides 17: 1243-1251.

Beaulieu P, Cardinal R, De Léan A, and Lambert C (1996) Direct chronotropic effects of atrial and C-type natriuretic peptides in anaesthetized dogs. $\mathrm{Br} J$ Pharmacol 118:1790-1796.

Beaulieu P, Cardinal R, Pagé P, Francoeur F, Tremblay J, and Lambert C (1997) Positive chronotropic and inotropic effects of C-type natriuretic peptide in dogs. Am J Physiol 273:H1933-H1940.

Chauhan SD, Nilsson H, Ahluwalia A, and Hobbs AJ (2003) Release of C-type natriuretic peptide accounts for the biological activity of endothelium-derived hyperpolarizing factor. Proc Natl Acad Sci USA 100:1426-1431.

Clavell AL, Stingo AJ, Wei CM, Heublein DM, and Burnett JC Jr (1993) C-type natriuretic peptide: a selective cardiovascular peptide. Am $J$ Physiol 264 R290-R295.

Furuya M, Tawaragi Y, Minamitake Y, Kitajima Y, Fuchimura K, Tanaka S, Minamino N, Kangawa K, and Matsuo H (1992) Structural requirements of C-type natriuretic peptide for elevation of cyclic GMP in cultured vascular smooth muscle cells. Biochem Biophys Res Commun 183:964-969.

He XL, Dukkipati A, Wang X, and Garcia KC (2005) A new paradigm for hormone recognition and allosteric receptor activation revealed from structural studies of NPR-C. Peptides 26:1035-1043.

Igaki T, Itoh H, Suga SI, Hama N, Ogawa Y, Komatsu Y, Yamashita J, Doi K, Chun TH, and Nakao K (1998) Effects of intravenously administered C-type natriuretic peptide in humans: comparison with atrial natriuretic peptide. Hypertens Res 21 : 7-13

Laederich MB and Horton WA (2010) Achondroplasia: pathogenesis and implications for future treatment. Curr Opin Pediatr 22:516-523.

Legeai-Mallet L (2016) C-type natriuretic peptide analog as therapy for achondroplasia. Endocr Dev 30:98-105.

Lopez M, L Garbers D, and Kuhn M (1997) The guanylyl cyclase-deficient mouse defines differential pathways of natriuretic peptide signaling. J Biol Chem 272 23064-23068.

Lorget F, Kaci N, Peng J, Benoist-Lasselin C, Mugniery E, Oppeneer T, Wendt DJ, Bell SM, Bullens S, Bunting S, et al. (2012) Evaluation of the therapeutic potential of a CNP analog in a Fgfr3 mouse model recapitulating achondroplasia. Am J Hum Genet 91:1108-1114.

Nakao K, Kuwahara K, Nishikimi T, Nakagawa Y, Kinoshita H, Minami T, Kuwabara Y, Yamada C, Yamada Y, Tokudome T, et al. (2017) Endothelium-derived C-type natriuretic peptide contributes to blood pressure regulation by maintaining endothelial integrity. Hypertension 69:286-296.

Oefner C, D'Arcy A, Hennig M, Winkler FK, and Dale GE (2000) Structure of human neutral endopeptidase (Neprilysin) complexed with phosphoramidon. J Mol Biol 296:341-349. 
Rau H, , Hersel U, , Sprogøe K, , Faltinger F, , Wegge T, and, and Cleemann F (2017), inventors, Ascendis Pharma Growth Disorders A/S, assignee. Controlledrelease CNP agonists with low NPR-C binding. International Patent Application WO 2017/118704. 2017 Jul 13.

Scotland RS, Ahluwalia A, and Hobbs AJ (2005) C-type natriuretic peptide in vascular physiology and disease. Pharmacol Ther 105:85-93.

Sprogøe K, , Hersel U, , Rau H, , Wegge T, , Faltinger F, , Cleemann F, , Kaluza N, , Bernhard A, , Buba A, and , and Woods T (2016) inventors, Ascendis Pharma Growth Disorders A/S, assignee. CNP Prodrugs. International Patent Application WO 2016/110577. 2016 Jul 14.

Watanabe Y, Nakajima K, Shimamori Y, and Fujimoto Y (1997) Comparison of the hydrolysis of the three types of natriuretic peptides by human kidney neutral endopeptidase 24.11. Biochem Mol Med 61:47-51.
Wendt DJ, Dvorak-Ewell M, Bullens S, Lorget F, Bell SM, Peng J, Castillo S, AoyagiScharber M, O’Neill CA, Krejci P, et al. (2015) Neutral endopeptidase-resistant C-type natriuretic peptide variant represents a new therapeutic approach for treatment of fibroblast growth factor receptor 3-related dwarfism. J Pharmacol Exp Ther 353:132-149.

Yasoda A, Kitamura H, Fujii T, Kondo E, Murao N, Miura M, Kanamoto N, Komatsu Y, Arai H, and Nakao K (2009) Systemic administration of C-type natriuretic peptide as a novel therapeutic strategy for skeletal dysplasias. Endocrinology 150: 3138-3144.

Address correspondence to: Dr. Vibeke Miller Breinholt, Tuborg Boulevard 12, DK-2900 Hellerup, Denmark. E-mail: vmb@ascendispharma.com 